\title{
TRANSCENDENTAL EXTENSIONS OF FIELD TOPOLOGIES ON COUNTABLE FIELDS
}

\author{
KLAUS-PETER PODEWSKI
}

\begin{abstract}
Let $\mathscr{T}$ be a field topology on a countable field $K$ and let $K(x)$ be a simple transcendental extension of $K$. Then there exists a field topology $\mathscr{T}^{\prime}$ for $K(x)$ such that $\mathscr{T}^{\prime} \uparrow K=\mathscr{T}$.
\end{abstract}

Let $K$ be a countable field and let $\mathfrak{A}$ be a fundamental system of neighborhoods at zero. We identify $K$ with the field of constant functions on $K$ and $K(x)$ with the field $R(K)$ of rational functions over $K$. If $D$ is a nonprincipal filter on $K$, then we define for each $U \in \mathfrak{A}, U^{D}$ to be the set of all $f \in R(K)$ with $\{r \mid f(r) \in U\} \in D$ and $\mathfrak{A}^{D}$ to be the set $\left\{U^{D} \mid U \in \mathfrak{A}\right\}$. $\mathfrak{A}^{D} 1 K=\mathfrak{A}$, and $\mathfrak{A}^{D}$ is a filter base. But $\mathfrak{A}^{D}$ is not necessarily a fundamental system of neighborhoods at zero of a field topology on $R(K)$.

1. Definition. A filter $D$ on $K$ is called $\mathfrak{A}$-generic, if for each $U \in \mathfrak{A}$ and for each $f \in R(K)$ there is a $V \in \mathfrak{A}$ such that $\{r \mid f(r) V \subset U\} \in D$.

2. THEOREM. If $D$ is a $\mathfrak{A}$-generic filter on $K$, then $\mathfrak{A}^{D}$ is a fundamental system on $R(K)$ such that $\mathfrak{U}^{D} 1 K=\mathfrak{U}$.

Proof. To see that $\mathfrak{A}^{D}$ defines a group topology on $R(K)$, let $U^{D} \in \mathfrak{A}^{D}$ be given. Since $\mathfrak{A}$ is a fundamental system there is a $V$ such that $V-V \subset U$. Suppose $f, g \in V^{D}$. Then $\{r \mid f(r) \in V\} \in D$ and $\{r \mid g(r) \in V\} \in D$. By

$$
\{r \mid f(r)-g(r) \in U\} \supset\{r \mid f(r) \in V\} \cap\{r \mid g(r) \in V\}
$$

we have that $f-g \in U^{D}$. Thus $V^{D}-V^{D} \subset U^{D}$.

By a similar argument, it can be seen that inversion and multiplication at zero are continuous. So it remains to show that multiplication is continuous everywhere. Let $f \in R(K)$ and $U^{D} \in \mathfrak{A}^{D}$ be given. Since $D$ is U-generic there is a $V$ such that $\{r \mid f(r) \cdot V \subset U\} \in D$. Suppose $g \in V^{D}$. Then $\{r \mid g(r) \in V\} \in D$.

$$
\{r \mid g(r) \cdot f(r) \in U\} \supset\{r \mid f(r) \cdot V \subset U\} \cap\{r \mid g(r) \in V\}
$$

and therefore $f \cdot g \in U^{D}$. Thus, multiplication is continuous everywhere.

Received by the editors March 2, 1972 and, in revised form, July 26, 1972.

AMS (MOS) subject classifications. Primary $12 \mathrm{~J} 99$.

(C) American Mathematical Society 1973 
Under certain conditions one can show that there exists a $\mathfrak{A}$-generic filter on $K$.

3. THEOREM. If $\mathfrak{A}$ is a countable fundamental system, then there is a $\mathfrak{U}$-generic filter $D$ on $K$.

Proof. If $\{0\} \in \mathfrak{A}$, then take $D$ to be the cofinite subsets of $K$. Otherwise let $\left(f_{n}\right)_{n \in \omega}$ be a well ordering of $R(K),\left(U_{m}\right)_{m \in \omega}$ a well ordering of $\mathfrak{A}$, and $\left(\left(n_{\alpha}, m_{\alpha}\right)\right)_{\alpha \in \omega}$ a well ordering of $\omega \times \omega$. By induction over $\alpha$ we choose nonempty open sets $0_{\alpha}$ as follows:

Let $0_{0}=K$. Assume that $0_{\alpha}$ has been chosen. Then there is a $r_{\alpha} \in 0_{\alpha}$ and a $V_{\alpha} \in \mathfrak{A}$, such that $f_{n_{\alpha}}\left(r_{\alpha}\right)$ is defined and $\left(f_{n_{\alpha}}\left(r_{\alpha}\right)+V_{\alpha}\right) V_{\alpha} \subset U_{m_{\alpha}}$. Because $f_{n_{\alpha}}$ is continuous at $r_{\alpha}$, there exists a $V_{\alpha}^{\prime} \in \mathfrak{A}$ such that $f_{n_{\alpha}}\left(r_{\alpha}+V_{\alpha}^{\prime}\right) \subset$ $f_{n_{\alpha}}\left(r_{\alpha}\right)+V_{\alpha}$. Define $0_{\alpha+1}$ to be $0_{\alpha} \cap\left(r_{\alpha}+V_{\alpha}^{\prime}\right)$. Thus $0_{\alpha}$ has been chosen for every $\alpha \in \omega$. By a straightforward argument, it can be seen that $\left\{0_{\alpha} \mid \alpha \in \omega\right\}$ is a base of a $\mathfrak{A}$-generic filter.

Thus we have shown that every field topology with countable basis on $K$, can be extended to a field topology on $K(x)$. Moreover, if the topology on $K$ is locally bounded, then the topology on $K(x)$ is locally bounded. To prove that every field topology on $K$ can be extended to a field topology on $K(x)$ we need the following Lemma:

4. Lemma. If $\mathfrak{A}$ is a fundamental system on $K$ and $U \in \mathfrak{A}$, then there is a countable fundamental system $\mathfrak{H}^{\prime} \subset \mathfrak{U}$ for a field topology with $U \in \mathfrak{A}^{\prime}$.

Proof. Let $\phi_{K}$ denote the set of functions $\varphi_{d}, \varphi_{c}, \varphi_{b}$, and $\varphi_{a}, a \in K$, defined by $\varphi_{d}(V)=V /(1-V), \varphi_{c}(V)=V \cdot V, \varphi_{b}(V)=V-V$ and $\varphi_{a}(V)=$ $a V$, for every subset $V$ of $K, 1 \notin V$. Let $\left(\varphi_{n}\right)_{n \in \omega}$ be a well ordering of $\phi_{K}$ and $\left(\left(n_{\alpha}, m_{\alpha}\right)\right)_{\alpha \in \omega}$ a well ordering of $\omega \times \omega$ such that $\alpha \geqq m_{\alpha}$.

By induction over $\alpha$ we choose sets $U_{\alpha} \in \mathfrak{A}$. Let $U_{0}=U$. Assume that $U_{0}, \cdots, U_{\alpha}$ have been chosen from $\mathfrak{A}$. Because $\mathfrak{A}$ is a fundamental system, there is a $V \in \mathfrak{A}$ such that $\varphi_{n_{\alpha}}(V) \subset U_{m_{\alpha}}$ and $V \subset U_{\alpha}$. Let $U_{\alpha+1}$ be such a $V$. Thus $U_{\alpha}$ is well defined for $\alpha \in \omega$. It is straightforward to see that $\left\{U_{\alpha} \mid \alpha \in \omega\right\}$ is a fundamental system, which contains $U$ and is a subset of $\mathfrak{A}$.

5. THEOREM. If $\mathscr{T}$ is a field topology on $K$, then there is a field topology $\mathscr{T}^{\prime}$ on $K(x)$ such that $\mathscr{T}^{\prime} 1 K=\mathscr{T}$.

Proof. Let $\mathfrak{A}$ be a fundamental system of neighborhoods at zero of $\mathscr{T}$. By Lemma 4 there is a set $\left\{\mathfrak{A}_{U} \mid U \in \mathfrak{U}\right\}$ of countable fundamental systems such that $\bigcup\left\{\mathfrak{A}_{U} \mid U \in \mathfrak{A}\right\}=\mathfrak{A}$. From Theorem 3, we see that for each $U \in \mathfrak{A}$ there is an $\mathfrak{A}_{U}$-generic filter $D_{U}$. By Theorem 2 we have that $\mathfrak{A}_{U}^{D^{\boldsymbol{J}}}$ is a fundamental system on $K(x)$ such that $\mathfrak{A}_{U}^{D_{v}} \mathfrak{} \mid K=\mathfrak{H}_{U}$. Hence, $\bigcup\left\{\mathfrak{U}_{U}^{D} v \mid U \in \mathfrak{A}\right\}$ is a subbase for a fundamental system $\mathfrak{A}^{*}$ on $K(x)$ such 
that $\mathfrak{U}^{*} \mid K=\mathfrak{A}$. If we take $\mathscr{T}^{\prime}$ to be the field topology defined by $\mathfrak{U}^{*}$, then $\mathscr{T}^{\prime}$ has the desired properties.

\section{REFERENCE}

1. L. A. Hinrichs, The existence of topologies on field extensions, Trans. Amer. Math. Soc. 113 (1964), 397-405. MR 30 \#91.

Institut für Mathematik, TeChNische Universität HanNover, Welfengarten 1, Hannover, West Germany 